
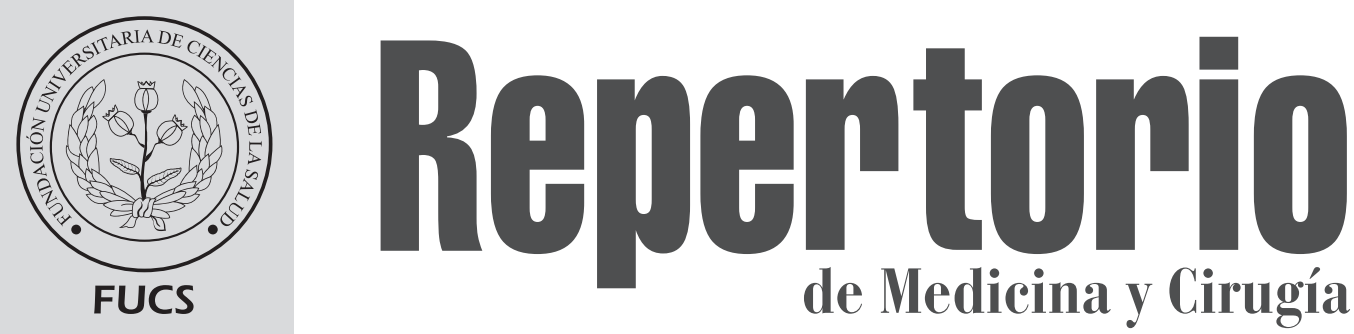

Vol. 280
No3. 2019

ISSN: 0121-7372 • ISSN electrónico: 2462-991X

Reporte de caso

\title{
Síndrome de Mayer-Rokitansky- Küster-Hauser asociado con malformación anorectal
}

Jesús Ángel Fernández MD Grisel María Marín Rincón MD

\section{Mayer-Rokitansky-Küster-Hauser syndrome associated with anorectal malformation}

${ }^{a}$ Unidad de Urología Pediátrica, Servicio de Cirugía Pediátrica, Servicio Autónomo Hospital Universitario de Maracaibo. Universidad del Zulia, Maracaibo, Venezuela.

${ }^{b}$ Servicio de Cirugía Pediátrica, Servicio Autónomo Hospital Universitario de Maracaibo. Universidad del Zulia, Maracaibo, Venezuela.

\section{R E S U M EN}

La agenesia vaginal es una entidad poco frecuente que puede presentarse en forma aislada o acompañada de otras anomalías congénitas. El desconocimiento de esta asociación puede retardar su diagnóstico, causar complicaciones indeseadas y comprometer el resultado del tratamiento. Se describen tres casos clínicos con agenesia vaginal y malformación anorrectal, cuyos diagnósticos no fueron realizados en el momento del nacimiento. Aunque la coexistencia de anomalías vaginales y anorectales sean poco frecuentes, los médicos responsables de su tratamiento deben ser conscientes de su existencia y practicar examen perineal cuidadoso.

Palabras clave: malformación anorrectal, agenesia vaginal, fistula vesicoperineal, rectovaginoplastia, anomalías del Müller.

(C) 2019 Fundación Universitaria de Ciencias de la Salud - FUCS Este es un artículo Open Access bajo la licencia CC BY-NC-ND (http://creativecommons.org/licenses/by-nc-nd/4.0/).

\author{
INFORMACIÓN DEL ARTÍCULO
}

Historia del artículo:

Fecha recibido: abril 25 de 2018

Fecha aceptado: marzo 27 de 2019
Autor para correspondencia.

Dr. Jesús Angel Fernández

jafernandezf@hotmail.com
DOI

10.31260/RepertMedCir.v28.n3.2019.962 


\section{A B S T R AC T}

Vaginal agenesis is a rare condition. It may exist in isolation or be associated with other congenital anomalies. A lack of awareness on this association may delay diagnosis, cause undesirable complications and compromise treatment outcomes. We describe three cases of vaginal agenesis associated with anorectal malformation which were not diagnosed at birth. Although the coexistence of vaginal and anorectal anomalies is uncommon, treating physicians should be aware of them and perform a detailed perineal examination.

Key words: malformation anorectal, vaginal agenesis, vesicoperineal fistula, rectovaginoplasty, Müllerian abnormalities

(C) 2019 Fundación Universitaria de Ciencias de la Salud - FUCS. This is an open access article under the CC BY-NC-ND license (http://creativecommons.org/licenses/by-nc-nd/4.0/).

\section{INTRODUCCIÓN}

La agenesia vaginal completa asociada con hipoplasia o agenesia uterina se conoce como síndrome de MayerRokitansky-Küester-Hauser (MRKH). Los criterios diagnósticos incluyen aplasia congénita de útero y vagina, fenotipo femenino, genitales externos y función ovárica normal y cariotipo $46 \mathrm{XX}$. Se cree que este síndrome afecta 1 de cada 4.000 a 5.000 niñas nacidas. ${ }^{1}$

Aunque puede tratarse de una agenesia uterovaginal aislada, con frecuencia se asocia con anomalías renales (40-60\%), esqueléticas $(10-12 \%)$, auditivas $(10 \%)$ y en un porcentaje menor con defectos cardiacos. ${ }^{2,3}$ Por otro lado, las anomalías anorectales afectan 1 de 3.500 nacimientos, siendo la forma más común las fistulas vestibulares. ${ }^{4} \mathrm{Si}$ bien se encuentra documentado que pacientes con alteraciones cloacales presentan malformaciones del aparato genital hasta en un $60 \%,{ }^{5}$ la presencia de fístulas recto-vestibulares o perineales no se ha descrito con el mismo detalle en la literatura. Gross mencionó un $0,4 \%$ de asociación entre atresia vaginal y malformaciones anorectales, ${ }^{6}$ mientras que Levitt y col. reportaron 1007 niñas, ocho con malformaciones anorectales, para una incidencia de $0.79 \% .^{7}$ El desconocimiento de esta asociación hace que la anomalía genital pase inadvertida ocasionando intervenciones secundarias con resultados poco deseados y un mayor número de complicaciones. ${ }^{8}$ El objetivo de este estudio es la presentación de 3 casos clínicos con malformaciones anorrectales y agenesia vaginal diagnosticadas en forma tardía y aportar nuestra experiencia en el manejo de las mismas.

\section{Caso 1}

Paciente a quien se realizó colostomía sigmoidea tipo Hartman en otro hospital el primer día de vida por haberse diagnosticado malformación anorrectal con fístula rectovestibular. Durante la anorrectoplastia efectuada a los 5 meses de edad, fueron descritos hallazgos compatibles con malformación tipo cloaca con un canal común de $2 \mathrm{~cm}$ y vagina estrecha, siendo sometida -según reporte quirúrgico- a anorrectovulvovaginoplastia con descenso en bloque del seno urogenital. A los 5 años de edad, la paciente consultó a nuestro hospital por no controlar el esfínter urinario. Al examen físico perineal se observó meato uretral desplazado dorsalmente con largo borde mucoso ventral, sin evidencia de introito vaginal; en su lugar presentó un orificio rodeado de mucosa de aproximadamente $5 \mathrm{~mm}$ de diámetro, a través del cual salía constantemente orina que erosionaba la piel vecina (figura 1). Una vez sospechada la agenesia vaginal se realizó ultrasonido pélvico que reportó ambos ovarios hipoplásicos. En la resonancia magnética de la pelvis no se visualizaron útero ni ovarios. La exploración laparoscópica identificó sólo ovario y trompa derecha, con ausencia de útero. El ultrasonido renal fue normal. La cistoscopia demostró fistula justo por encima de orificio uretral interno, que comunicaba con el orificio perineal descrito. Luego de diagnosticar fístula vesico-perineal, se ha intentado cierre infructuoso de la misma en 2 oportunidades tanto a través de la vejiga como del periné. La continencia fecal es normal. En la actualidad se programará para corrección quirúrgica de dicha fístula más vaginoplastia.

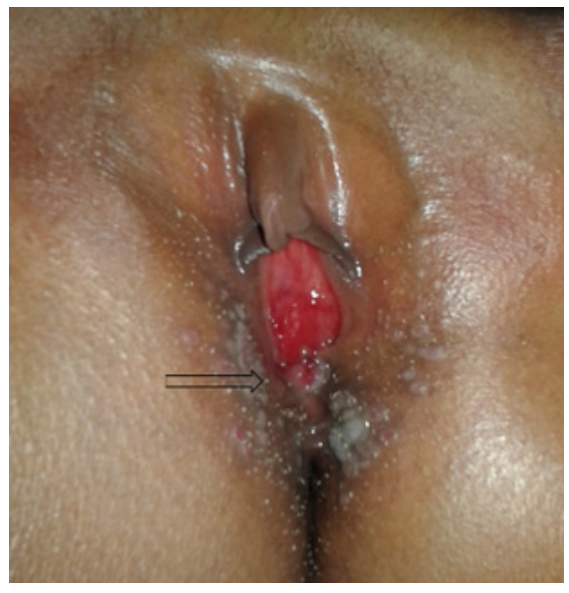

Figura 1. Postoperatorio de paciente con anomalía anorrectal y agenesia vaginal. Meato uretral con largo borde mucoso ventral. No existe introito vaginal ${ }_{i}$ en su lugar orificio mucoso (flecha) con salida constante de orina, que causa dermatitis local. 


\section{Caso 2}

Paciente quien consultó por haberse diagnosticado agenesia anorrectal y fístula rectovestibular, realizando colostomía sigmoidea tipo Hartman el primer día de vida. Al examen perineal minucioso en el sexto mes la apariencia de los labios mayores y menores fue normal, pero no se encontró introito vaginal en el área del vestíbulo. De dos aberturas en el vestíbulo, la anterior correspondió a la uretra, mientras la posterior condujo al recto. El ultrasonido pélvico fue reportado normal. La laparoscopia demostró ovario y trompa derecha normales unidos a un hemiútero rudimentario. El ovario izquierdo con una trompa rudimentaria no tenía comunicación con el útero. A los 10 meses de vida se realizó anorrectoplastia con rectovaginoplastia; el extremo distal del recto se dejó in situ al tiempo que el proximal se seccionó por debajo de la reflexión peritoneal y se descendió hasta el ano (figura 2).


Figura 2. A: el recto distal dejado in situ se secciona a nivel de la reflexión peritoneal, al tiempo que el recto proximal es descendido hasta el periné dentro del complejo muscular (B).

Un año más tarde se incidió el reborde mucoso de la línea media de la desembocadura original del recto en el vestíbulo (horquilla posterior), para ampliar el introito vaginal. Luego de 5 años de seguimiento, el aspecto estético de la vulva es excelente; introito vulvar amplio y vagina con profundidad de $4,5 \mathrm{~cm}$ (figura 3). La niña presenta control fecal voluntario con estreñimiento.

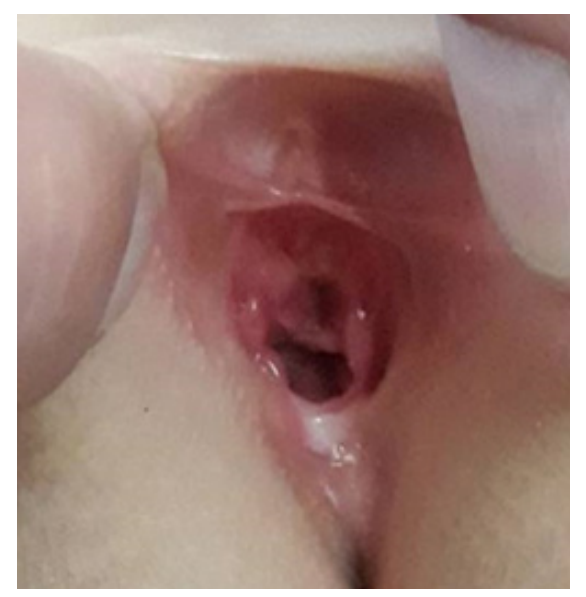

Figura 3. Postoperatorio de rectovaginoplastia en paciente con malformación anorrectal y fístula rectovestibular. Aspecto estético satisfactorio, introito vulvar amplio.

\section{Caso 3}

Paciente a quien se realizó colostomía sigmoidea descendente a dos bocas en las primeras 24 horas de vida por presentar malformación anorrectal tipo cloaca. El examen físico permitió observar un orificio perineal único. El surco interglúteo era poco pronunciado, músculos glúteos poco desarrollados sin fóvea anal. En la endoscopia se evidenció seno urogenital con canal de $2 \mathrm{~cm}$ sin observar cuello uterino. La ecografía renal mostró riñón único izquierdo y el ecograma pélvico no evidenció genitales internos (ovarios ni útero). Ecocardiograma normal. La radiología simple de tórax reveló hemivertebras en las primeras dorsales. El cariotipo fue 46XX. Durante la anorrectovulvovaginoplastia realizada a los 3 años de edad, no se evidenció vagina (figura 4).

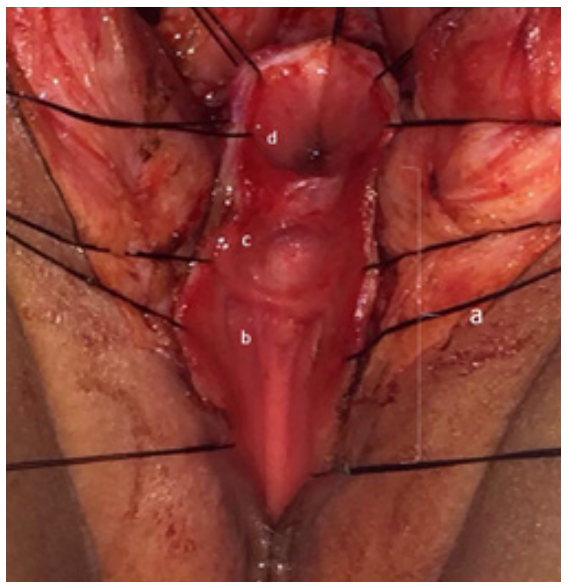

Figura 4. Transoperatorio de paciente con anomalía anorrectal tipo cloaca y agenesia vaginal, posterior a incisión del canal común de aproximadamente $2,5 \mathrm{~cm}$ de longitud (a), se observa el meato uretral (b), la fóvea vaginal (c) y el ano (d).

Se descendió el seno urogenital, se efectuó rectovaginoplastia con recto distal y se descendió el recto proximal. El aspecto estético actual es satisfactorio, aunque el meato uretral es discretamente hipospádico, el introito vulvar es amplio y la longitud de la rectovagina es de 3,5 cm de longitud (figura 5).

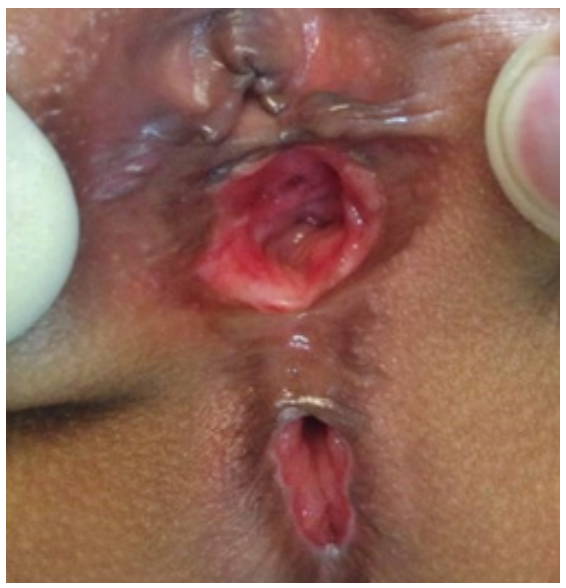

Figura 5. Postoperatorio de paciente con anomalía anorrectal tipo cloaca y agenesia vaginal, descenso del seno urogenital con rectovaginoplastia. Aspecto estético satisfactorio, meato uretral discretamente hipospádico, introito vulvar amplio. 
La incidencia de anomalías del Müller en pacientes con fístulas rectovestibulares probablemente esté subestimada en neonatos y lactantes. Aunque la identificación de ano imperforado es común, la atresia de la vagina es más difícil de detectar. Al igual que ocurrió en nuestras tres pacientes, en quienes el diagnóstico fue realizado a los 6 meses de vida en dos de ellas y a los 5 años de edad en la otra, existe evidencia en la literatura donde esta condición pasa inadvertida en las primeras etapas de la vida. Más lamentable aún, en ocasiones se realiza anoplastia sin que se diagnostique la anomalía uterovaginal. En otras oportunidades es durante el procedimiento quirúrgico que se hace evidente la malformación genital. Lo anterior conlleva a reintervenciones quirúrgicas, peores resultados y mayor número de complicaciones. En el primero de los casos reportados, en quien se realizó el diagnóstico transoperatorio de malformación tipo cloaca, sospechamos que fue descendida la pared posterior de la vejiga, provocando una fístula vesicoperineal de muy difícil corrección.

El manejo inicial de las niñas con agenesia anorrectal y fístula rectovestibular debe comenzar por un examen cuidadoso del periné en búsqueda de tres orificios (uretra, vagina y fístula rectal). En caso de dudas hay que practicar procedimientos endoscópicos combinados con estudios imagenológicos, ya sean invasivos o no. Estos incluyen radiografía pélvica, ultrasonido pélvico y espinal, y resonancia magnética. El ultrasonido de la pelvis en neonatos tiene muchas limitaciones por la presencia de asas intestinales llenas de líquido que comprometen la visualización adecuada de los órganos pélvicos. La resonancia magnética es una excelente herramienta para observar la pelvis femenina sin exposición a radiaciones, aunque requiere anestesia general. Sarin y col. recomiendan realizar endoscopia y resonancia magnética en todos los casos con malformaciones anorectales y agenesia uterovaginal, basados en la excelente resolución de estos estudios. ${ }^{9,10}$ Cuando la anatomía no es muy clara, Jarboe y col. han descrito una técnica para pacientes con anomalías cloacales, en quienes colocan contraste dentro de los orificios pélvicos y realizan resonancia con reconstrucción. ${ }^{11} \mathrm{La}$ radiografía de la pelvis suele servir para determinar el radio del sacro, lo cual es útil en términos de pronóstico de continencia fecal.

La laparoscopia además de la evaluación completa de los genitales internos permite examinar el colon distal y en un momento dado ayuda a elegir el sitio para la colostomía. ${ }^{10,12} \mathrm{El}$ segmento del colon donde se realice la colostomía es importante para no comprometer su uso futuro en la vaginoplastia. Kartikey y col. recomiendan una transversa que permita mayor movilización del colon descendente para la reconstrucción vaginal. ${ }^{10}$

El tratamiento quirúrgico definitivo plantea algunas consideraciones en cuanto a la edad en que debe practicarse y la reconstrucción a realizar. La corrección temprana del defecto vaginal contribuye a la sensación de bienestar de la paciente y ayuda a evitar el trauma de una cirugía correctiva tardía.
Wester y col. presentaron una serie de pacientes, la mayoría de las cuales fueron sometidas a reconstrucción simultánea de ano y vagina en el primer año de vida. ${ }^{13}$ Esto ofrece la ventaja de prevenir por un lado el trauma psicológico y por otro evita la necesidad de vaginoplastia a través del periné cicatrizado posterior a la anorectoplastia. ${ }^{14}$ Esta reconstrucción simultánea ha sido la estrategia en la mayoría de las publicaciones recientes. $7,9,15,16$

Con respecto a la técnica de reconstrucción vaginal, la rectovaginoplastia ha sido la más empleada. ${ }^{6,8,9,13-15,17}$ Levitt y col. usaron la parte distal del recto como neovagina, descendiendo el recto a través de anorrectoplastia sagital posterior. ${ }^{18}$ Esto evita la tediosa separación del recto de la uretra, disminuyendo el riesgo de vejiga neurogénica, trauma uretral y compromiso de continencia fecal. Otras modalidades es la utilización del íleon terminal en el reemplazo de la vagina. ${ }^{8}$ No existe mucha información en mujeres sexualmente activas con relación a la función de la neovagina para propósitos sexuales, por lo que ha sido difícil evaluar a largo plazo. En este sentido, Hensle y col. ${ }^{19}$ reportaron que $78 \%$ de 36 pacientes manifestaron satisfacción sexual después de vaginoplastia con intestino, sin embargo $95 \%$ de ellas usaban duchas frecuentes y 56\% toallas, lo cual ilustra que la producción de moco es un problema común en este tipo de vaginoplastias. También hay reportes individuales sobre relaciones sexuales difíciles e insatisfactorias. ${ }^{18}$

En la capacidad eventual de la niña para procrear, la agenesia mülleriana presenta serias implicaciones, por lo que el diagnóstico precoz de esta afección permite que los problemas y las preocupaciones psicológicas se evalúen con atención y se aborden en la vida temprana de la niña. ${ }^{13} \mathrm{El}$ tratamiento de los cuernos uterinos ha sido controversial en pacientes que presentan remanentes permeables con endometrio normal, reportado del 2 al $7 \%$ de los casos. ${ }^{20}$ Las opciones terapéuticas incluyen la escisión quirúrgica, la anastomosis durante la creación de la neovagina o mantener una conducta expectante. Este endometrio secretor se asocia con complicaciones tardías por hematometra y dolor pélvico cíclico una vez iniciada la pubertad. ${ }^{21}$ Si logra determinarse la permeabilidad de los cuerpos uterinos durante la cirugía, podría realizarse la anastomosis de estos a la neovagina al momento de la vaginoplastia, sin embargo este procedimiento no está exento de complicaciones y no es inusual la necesidad de escisión quirúrgica futura de los cuerpos uterinos. La decisión de anastomosarlos a la vagina se hace más compleja por los registros históricos de infecciones uterinas ascendentes favorecidas por la ausencia de cuello. Aunque se reportan embarazos a término en estas pacientes, con frecuencia conducen a abortos espontáneos. ${ }^{22}$ En pacientes con útero ausente o remanentes completamente fibrosos no es necesario el reimplante, por lo que deben resecarse, a pesar de que algunos padres desean la anastomosis ante la esperanza de que las niñas puedan tener periodos menstruales durante la adolescencia. 


\section{CONCLUSIONES}

Los especialistas responsables del tratamiento de niñas con malformaciones anorectales y del Müller deben ser conscientes de la existencia de esta asociación. El examen perineal cuidadoso permitirá realizar un diagnóstico precoz en la mayoría de las pacientes, lo que orientará sobre estudios imagenológicos y la planificación y realización de procedimientos quirúrgicos específicos en el momento adecuado para evitar complicaciones quirúrgicas importantes e irreversibles, y mitigar el impacto psicológico en la paciente y la familia.

\section{CONFLICTO DE INTERÉS}

Los autores declaran que no tienen conflicto de interés potenciales relacionados con los contenidos de este artículo.

\section{REFERENCIAS}

1. Meyers R. Congenital anomalies of the vagina and their reconstruction. Clin Obstet Gynecol. 1997; 40(1):168-80.

2. Morcel K, Camborieux L. Guerrier D. Programme de Recherches sur les Aplasies Mülleriennes, The Mayer-Rokitansky-KüsterHauser (MRKH) syndrome. Orphanet J Rare Dis. 2007; 2:13-21. doi: 10.1186/1750-1172-2-13

3. Edmonds D. Congenital malformations of the genital tract and their management. Best Pract Res Clin Obstet Gynaecol. 2003; 17(1):19-40.

4. Peña A. Anorectal malformations. Semin Pediatr Surg. 1995; $4(1): 35-47$.

5. Levitt $M$, Peña A. Cloacal malformations: lessons learned from 490 cases. Semin Pediatr Surg. 2010; 19(2): 128-38. doi: 10.1053/j. sempedsurg.2009.11.012.

6. Cohn B, Murphy D. Imperforate anus with agenesis of the vagina. Ann Surg. 1956; 143(3): 430-2.

7. Levitt M, Stein D, Peña A. Rectovestibular fistula with absent vagina: a unique anorectal malformation. J Pediatr Surg. 1998; 33(7):986-9.

8. Levitt M, Bischoff A, Breech L, Peña A. Rectovestibular fistulararely recognized associated gynecologic anomalies. J Pediatr Surg. 2009;44(6):1261-7. doi: 10.1016/j.jpedsurg.2009.02.046.

9. Sarin Y, Sinha A. Two orifices in perineum of a girl with imperforate anus: possibility of uterovaginal agensis associated with retovestibular fistula. J Pediatric Surgery. 2002;37(8): 121719.

10. Pandya K, Koga H, Okawada M, Coran A, Yamataka A, Teitelbaum D. Vaginal anomalies and atresia associated with imperforate anus: Diagnosis and surgical management. J Pediatr Surg. 2015;50(3): 431-37. doi: 10.1016/j.jpedsurg.2014.07.010.
11. Jarboe M, Teitelbaum D, Dillman J. Combined 3D rotational fluoroscopic-MRI cloacagram procedure defines luminal and extraluminal pelvic anatomy prior to surgical reconstruction of cloacal and other complex pelvic malformations. Pediatr Surg Int. 2012; 28(8):757-63. doi: 10.1007/s00383-012-3122-6.

12. Teo X, Narasimhan K, Chua J. Müllerian agenesis in the presence of anorectal malformations in female newborns: a diagnostic challenge. Singapore Med J. 2015; 56(5): e82-4. doi: 10.11622/ smedj.2015079.

13. Wester T, Tovar J, Rintala R. Vaginal agenesis or distal vaginal atresia associated with anorectal malformations. J Pediatr Surg. 2012; 47(3):571-6. doi: 10.1016/j.jpedsurg.2011.09.040.

14. Jayaswal S, Dhende N, Mane S. Anorectal Malformation with Mayer-Rokitansky Syndrome - A Rare Association. Bombay Hospital Journal. 2008;50(1):104-6.

15. Günsar C, Genç A, Şencan A, Dağlar Z, Alparslan O, Mir E. MURCS association and rectovestibular fistula: case report of a patient treated with one-stage posterior sagittal anorectoplasty and sigmoid loop vaginoplasty. J Pediatr Surg. 2003;38(2):262-4. doi: 10.1053/jpsu.2003.50060

16. Patankar J, Mali V, Yashpal R, Neo G. Prabhakaran K. Anorectal malformation with congenital absence of vagina: a case report and review of the literature. Pediatr Surg Int. 2004;20(4):295-7. doi: 10.1007/s00383-003-1130-2

17. Foley S, Morley G. Care and counseling of the patient with vaginal agenesis. The Female Patient. 1992;17:73-80.

18. Wang S, Lang J, Zhu L. Mayer-Rokitansky-Küester-Hauser (MRKH) syndrome with rectovestibular fistula and imperforate anus. Eur J Obstet Gynecol Reprod Biol. 2010;153(1): 77-80. doi: 10.1016/j.ejogrb.2010.07.003.

19. Hensle T, Shabsigh A, Shabsigh R, Reiley E, Meyer-Bahlburg H. Sexual function following bowel vaginoplasty. J Urol. 2006;175(6):2283-6. doi: 10.1016/S0022-5347(06)00337-5

20. ACOG Committee Opinion. Number 274, July 2002. Nonsurgical diagnosis and management of vaginal agenesis. Committee on Adolescent Health Care. Obstet Gynecol. 2002;100(1):213-6.

21. Will M, Smorgick N, Quint E, Hussain H, Smith Y. Uterine remnants and pelvic pain in females with Mayer-RokitanskyKüster-Hauser syndrome. J Pediatr Adolesc Gynecol. 2013; 26(3):199-202.

22. Acién P, Acién M, Quereda F, Santoyo T. Cervicovaginal agenesis: spontaneous gestation at term after previous reimplantation of the uterine corpus in a neovagina: case report. Hum Reprod. 2008;23(3):548-53. doi: 10.1093/humrep/dem420. 